YITP-99-23

hep-th/9904118

\title{
Moduli Space of Vacua of Supersymmetric Chern-Simons Theories and Type IIB Branes
}

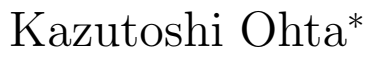 \\ Yukawa Institute for Theoretical Physics, Kyoto University, Kyoto 606-8502, Japan
}

\begin{abstract}
We study the correspondence between the moduli space of vacua of three-dimensional supersymmetric Yang-Mills (Maxwell) Chern-Simons theories and brane configurations with $(p, q) 5$-brane. For Coulomb branches, the number of the massless adjoint scalar fields in various supersymmetric theories exactly coincides with the number of the freely moving directions of D3-branes stretched between two 5-branes. When we include a matter superfield into the supersymmetric Chern-Simons theory two distinct symmetric and asymmetric phase appear. The symmetric phase is peculiar to this Chern-Simons Higgs system. We find the corresponding brane configuration for these phases. We also identify the stringy counterpart of the topological vortex state in the asymmetric phase.
\end{abstract}

\footnotetext{
*e-mail address: kohta@yukawa.kyoto-u.ac.jp
} 


\section{Introduction}

Recent developments in string theory are understandings of the correspondence between the brane dynamics and the physics of gauge theories in various dimensions. Hanany and Witten have first pointed out [1] that $N=4$ supersymmetric gauge theory in threedimensions is realized as worldvolume effective theory on D3-branes stretched between two NS5-branes and the "mirror symmetry" can be understand as a consequence of $S L(2, \mathbf{Z})$ duality of Type IIB superstring theory.

Their construction is extended to different numbers of supersymmetries [2, 3] and gauge theory in various dimensions (for a review see «, 4]). The advantage of this approach is that we can geometrically analyze the structure of the moduli spaces of vacua as the configuration space of branes. The geometrical construction of moduli space make easy to classify possible supersymmetric vacuum.

From the point of view of brane solutions in Type IIB superstring theory, the threedimensional Hanany-Witten type configuration is generalized to the case including rotated 5 -branes at arbitrary angles [6]. It contain the interesting 5 -brane solution preserving 3/16 of supersymmetry. This means that in three-dimensions we have $N=3$ supersymmetric theory. One of the realization of the three-dimensional $N=3$ supersymmetry is obtained by adding the Chern-Simons term [7, 8, 9]. So the brane configuration with 3/16 supersymmetry can be associated with three-dimensional Chern-Simons theory.

Moreover, the systematic classification of possible Hanany-Witten type configuration with various supersymmetries has been studied in ref. [10. Since the Type IIB brane configuration with $3 / 16$ supersymmetry contains a $(p, q) 5$-brane which is a bound state of NS5 and D5-brane, it is reasonable to consider that the replacement of one of the NS5branes with the $(p, q) 5$-brane is related to adding the Chern-Simons interaction. Under this identification, it is found that the vacuum structure of Maxwell Chern-Simons theory is consistent with the brane picture and a "mirror symmetry" is similarly realized by Type IIB S-duality. This "mirror symmetry" in supersymmetric Chern-Simons theory is recently confirmed by using a generalized Fourier transformation in ref. [11].

In this paper, we investigate the correspondence between the $(p, q) 5$-brane configuration introduced by ref. [10] and Yang-Mills (Maxwell) Chern-Simons theories in de- 
tail. More specifically, we construct a explicit Lagrangian for the supersymmetric ChernSimons theories and identify vevs of emergent fields with moduli parameters of Type IIB branes. This identification gives us the knowledge to understand the complicated dynamics of three-dimensional massive gauge theories and the dynamics of Type IIB branes.

This paper is organized as follows: In section 2 we briefly review the construction of the brane configurations with $(p . q) 5$-brane. These configurations in Type IIB theory are reduced from M5-brane configurations in M-theory with various supersymmetries. We give all possible Hanany-Witten type configuration with $N \geq 2$ supersymmetry. In section 3, we see how to describe the Coulomb branch by vevs of adjoint scalars in supersymmetric Chern-Simons theory. The number of the adjoint scalar fields corresponds to the number of freely moving directions of D3-branes. Finally, we consider the vacuum of Maxwell ChernSimons theory with matter superfields. In this model, there are two distinct vacua. One of vacua, which is called as a symmetric phase, exists on in the Maxwell Chern-Simons Higgs system. We can find the brane configuration of the symmetric phase. We also identify the topological vortex in the asymmetric phase as a bound state of strings.

\section{Supersymmetric Configuration with $(p, q)$ 5-brane}

In this section we briefly review the construction of the brane configuration with $(p, q) 5$ brane.

The brane configurations in Type IIB string theory which are given by Hanany and Witten [四] describe three dimensional supersymmetric gauge theories. In this configuration D3-branes are suspended between two parallel NS5-branes and the $3+1$ dimensional worldvolume of D3-brane is compactified on a finite line segment. We denote the extended directions of two NS5-branes and $N_{c}$ D3-branes as the following symbols:

$$
\begin{aligned}
& 2 \times \mathrm{NS} 5:(012345), \\
& N_{c} \times \mathrm{D} 3:(012|6|),
\end{aligned}
$$

where the numbers in the parenthesis are the worldvolume directions and the vertical lines on both side of 6 mean that the worldvolume of D3-branes are restricted to finite interval in the $x^{6}$-direction. The "left" NS5-brane is located at $x^{6}=-L / 2$ and the "right" is at 
$x^{6}=L / 2$.

The low-energy effective theory on $N_{c}$ parallel D3-branes is $3+1$ dimensional $S U\left(N_{c}\right)$ supersymmetric Yang-Mills theory in the limit that string scale $l_{s}$ goes to zero, but this compactification on the line reduces the D3-brane worldvolume theory to $2+1$ dimensions. Upon this Kaluza-Klein reduction on the line segment we find the gauge coupling constant $g$ of this worldvolume theory is given by

$$
\frac{1}{g^{2}}=\frac{L}{g_{s}}
$$

where $g_{s}$ is a string coupling constant of Type IIB theory and $L$ is a length of the line segment, namely, a distance between two NS5-branes in the $x^{6}$-direction.

In this configuration the presence of these two kinds of branes preserves 8 of 32 supercharges of Type IIB string theory since each kind of brane preserves half of supercharges. Therefore we have $N=4$ supersymmetry in $2+1$ dimensions.

To add matter in the fundamental representation of the gauge group we can introduce $N_{f}$ D5-branes, which have the worldvolume:

$$
N_{f} \times \mathrm{D} 5 \quad: \quad(012789)
$$

without breaking any supersymmetries. The matter hypermultiplets come from strings stretched between the $N_{c}$ suspended D3-branes and the $N_{f}$ D5-branes. So $N_{f}$ represents the number of flavors.

In addition to these types of branes there also exists the $(p, q) 5$-brane which is a bound state of NS5- and D5-branes. One of the extension of the above setup is replacement of the right NS5-brane in the Hanany-Witten configuration with this $(p, q) 5$-brane. However, if we simply substitute the $(p, q) 5$-brane for the right NS5-brane and the left NS5-brane and the right $(p, q) 5$-brane is parallel then all supersymmetry is broken. To preserve some of the supercharges we must rotate the $(p, q) 5$-brane by some angles.

In order to count the residual supercharges we now lift the above Type IIB configuration to M5- and M2-brane configurations in M-theory. T-duality in the $x^{2}$-direction maps the NS5-brane and the D5-brane into the NS5-brane and the D4-brane in Type IIA theory, respectively. By lifting to M-theory these objects become M5-branes. The NS5-brane corresponds to the M5-brane wrapping on the $x^{2}$-direction and the D5-brane 
corresponds to the M5-brane wrapping on the 11 th-direction $x^{10}$. So two cycles of the torus $T^{2}$ with coordinates $\left(x^{2}, x^{10}\right)$ are related to NSNS-charge and RR-charge in Type IIB theory. Similarly, $(p, q) 5$-brane in Type IIB theory is also unified as a M5-brane which is obliquely wrapping on the torus $T^{2}$ at some angle $\theta$. This angle and charges of the $(p, q) 5$-brane are related each other as follows

$$
\tan \theta=\frac{2 \pi R_{10} p}{2 \pi R_{2} q}=g_{s} \frac{p}{q}
$$

where $R_{2}$ and $R_{10}$ are radii of the torus $T^{2}$.

Using the above procedure D3-branes in Type IIB theory become M2-branes stretched between two M5-branes in M-theory. In this situation the right M5-brane tilted by the angle $\theta$ can be rotated by more three angles in the configuration space of M-theory. So, we have a general configuration in M-theory:

$$
\begin{aligned}
\mathrm{M} 5 & :(012345) \\
N_{c} \times \mathrm{M} 2 & :(01|6|) \\
\mathrm{M} 5^{\prime} & :\left(01\left[\begin{array}{c}
2 \\
10
\end{array}\right]_{\theta}\left[\begin{array}{l}
3 \\
7
\end{array}\right]_{\psi}\left[\begin{array}{l}
4 \\
8
\end{array}\right]_{\varphi}\left[\begin{array}{l}
5 \\
9
\end{array}\right]_{\rho}\right),
\end{aligned}
$$

where the symbol $\left[\begin{array}{l}\mu \\ \nu\end{array}\right]_{\theta}$ means that the extended direction of the M5'-brane are rotated by the angle $\theta$ in the $\left(x^{\mu}, x^{\nu}\right)$-space. If the angle $\theta$ is zero $\mathrm{M} 5^{\prime}$-brane become a rotated NS5-brane in Type IIB configuration space. On the other hand, the case of $\theta=\pi / 2$ corresponds to a D5-brane.

The presence of these branes imposes the constrains on the 11-dimensional Killing spinors $\epsilon$ [12]

$$
\begin{aligned}
\text { M5 } & : \Gamma_{012345} \epsilon=\epsilon, \\
\text { M2 } & : \Gamma_{016} \epsilon=\epsilon, \\
\text { M5 }^{\prime}: & R \Gamma_{012345} R^{-1} \epsilon=\epsilon,
\end{aligned}
$$

where $R$ is the rotation matrix in the spinor representation and give by

$$
R=\exp \left\{\frac{\theta}{2} \Gamma_{2,10}+\frac{\psi}{2} \Gamma_{37}+\frac{\varphi}{2} \Gamma_{48}+\frac{\rho}{2} \Gamma_{59}\right\} .
$$

The numbers of the remaining supersymmetry are obtained by solving eqs. (2.3)-(2.5) simultaneously. All possible solutions are completely classified in ref. [10 by setting some 
relations between four angles and we have $1 / 16,1 / 8,3 / 16$ and $1 / 4$ of the original 32 supercharges. These fractions of supersymmetry correspond to $N=1,2,3,4$ supersymmetries, respectively, in $2+1$ dimensions.

In present paper we treat only the configuration for $N \geq 2$ supersymmetry. This is because quantum corrections of higher supersymmetric is suppressed rather than the $N=1$ case and it is not so complicated to find the correspondence to the branelogy.

$N \geq 2$ supersymmetric configurations are divided into the following two cases. One is the NS5 configuration with $N=2,4$ supersymmetry where the right 5-brane is the NS5brane. The other is the configuration including $(p, q) 5$-brane with $N=2,3$.

We first consider the NS5 configuration. For $N=4$ configuration we must set all angles to zero, $\theta=\psi=\varphi=\rho=0$. The right M5'-brane is a NS5-brane in Type IIB theory, which is parallel to the left NS5-brane. This is nothing but the Hanany-Witten configuration. As we mentioned above, this configuration describes $N=4 S U\left(N_{c}\right)$ supersymmetric YangMills theory. This theory includes three real scalar fields in adjoint representation. Vevs of these adjoint scalars and scalars dual to the gauge fields parametrize the Coulomb branch. On the other hand, D3-branes in the brane configuration can freely move along the $\left(x^{3}, x^{4}, x^{5}\right)$-space. These positions of D3-branes on NS5-brane correspond to the vevs of real adjoint scalars. In addition the $S O(3)$ rotation group in the $\left(x^{3}, x^{4}, x^{5}\right)$ can be identified with a part of the $R$-symmetry $S U(2)_{V}$ of $N=4$ supersymmetry algebra in three dimensions. The other part of the $R$-symmetry $S U(2)_{H}$ corresponds to rotations in the $\left(x^{7}, x^{8}, x^{9}\right)$-space.

The $N=2$ NS5 configuration is obtained by a rotation of the right NS5-brane. In this rotation we must set same values on two pairs of angles. Since $\theta$ is zero in NS5 configuration one of our choice for angles is $\theta=\rho=0$ and $\varphi=\psi \neq 0$. The special case of $\varphi=\psi=\pi / 2$ is investigated in ref. [2] and this brane configuration space well describes the moduli space of vacua of $N=2$ supersymmetric Yang-Mills theory. The Coulomb branch of this theory is parametrized by vevs of one real massless adjoint scalar and dual gauge fields. In this configuration D3-branes can not move in the $\left(x^{3}, x^{4}\right)$-space any longer since two NS5-branes are completely twisted in that space. Then the vev of real adjoint scalar corresponds to the positions of D3-branes in $x^{5}$-direction. For the general value of $\psi$ this rotation angle is related to a mass of two real adjoint scalars [13, 14], which originally 
belong to the $N=4$ vector multiplet. Therefore we can identify the rotation of NS5-brane with the supplement of the mass term of two adjoint scalars into $N=4$ theory. Actually, this brane rotation breaks the $S U(2)_{V} \times S U(2)_{H}$ rotation symmetry to $S O(2) \simeq U(1)_{R}$, which coincide with the $N=2 R$-symmetry group.

Let us next mention about the configuration with $(p, q) 5$-brane. This configuration is realized by setting $\theta \neq 0$.

A maximal supersymmetric configuration with $(p, q)$-brane is the case of $\theta=\psi=\varphi=$ $-\rho$. This configuration preserves $N=3$ supersymmetry in $2+1$ dimensions. Since the all rotation angles are same except for the signature, the rotation symmetry $S U(2)_{V} \times S U(2)_{H}$ in the $\left(x^{3}, x^{4}, x^{5}\right)$ - and $\left(x^{7}, x^{8}, x^{9}\right)$-spaces breaks to a diagonal $S U(2)_{D}$. This rotational symmetry $S U(2)_{D}$ can be considered as the $R$-symmetry of the three-dimensional $N=3$ supersymmetry algebra.

$N=2$ configuration is given by setting $\rho=-\theta \neq \varphi=\psi$, that is, two different sets of same angles. If we take $\theta=\rho=0$, then we have the $N=2$ NS5 configuration as mentioned above. Similarly, we can choose as $\varphi=\psi=0$. In this case the right 5-brane is $(p, q) 5$-brane but still $N=2$ supersymmetric.

It is field theoretically shown that the three-dimensional $N=3$ maximal supersymmetric system can be constructed by adding Chern-Simons terms [7, 8, 9]. So one expect [6] that the worldvolume effective theory on this $2+1$-dimensional intersection of rotated 5branes is gauge theories with Chern-Simons terms. In fact, since there exists a non-trivial vev of axion (RR-scalar) field $C_{0}$ around $(p, q) 5$-brane in Type IIB theory, the coupling $\int C_{0} F \wedge F$ on the D3-brane induces the Chern-Simons term with coupling $\kappa=p / q$ [10].

In the following section, we investigate the correspondence between these supersymmetric configurations with the $(p, q) 5$-brane and the moduli space of vacua of ChernSimons theories.

\section{Coulomb Branches of The Non-Abelian Theories}




\section{1 $N=3$ supersymmetric Yang-Mills Chern-Simons theory}

We first begin with the $N=3$ configuration. The configuration in Type IIB theory is written as follows in the notation of the previous section:

$$
\begin{aligned}
\mathrm{NS} 5 & :(012345) \\
N_{c} \times \mathrm{D} 3 & :(012|6|) \\
(p, q) 5 & :\left(01\left[\begin{array}{l}
3 \\
7
\end{array}\right]_{\theta}\left[\begin{array}{l}
4 \\
8
\end{array}\right]_{\theta}\left[\begin{array}{l}
5 \\
9
\end{array}\right]_{-\theta}\right),
\end{aligned}
$$

where $\theta=\tan ^{-1}\left(g_{s} \frac{p}{q}\right)$. There originally exists $S U\left(N_{c}\right)$ Yang-Mills gauge theory on the D3-branes. As we have explained, the right $(p, q)$ 5-brane provides the Chern-Simons term into the Yang-Mills theory. So we have the Yang-Mills Chern-Simons theory as the effective theory on this configuration. The coupling constant of Chern-Simons term is given by $\kappa=p / q$. However, the coupling constant of the non-Abelian Chern-Simons theory should be a integer in order to preserve gauge invariance under a large transformation. Therefore we set the charges of $(p, q) 5$-brane as $(p, q)=(n, 1)$.

$N=3$ supersymmetric field theory in three dimensions has the $S U(2)_{D} R$-symmetry. The vector multiplet contains one spin 1 massive vector field $A_{\mu}$, three spin $1 / 2$ spinors $\lambda_{i}$, three spin 0 real adjoint fields $X_{i}$ and one spin $-1 / 2$ spinor $\chi$. Three spinors and adjoint scalars form a triplet under $S U(2)_{D}$. The bosonic part of the Lagrangian of this $N=3$ supersymmetric Yang-Mills Chern-Simons theory is given by 9]

$$
\begin{aligned}
\mathcal{L}_{\mathrm{B}}= & -\frac{1}{g^{2}} \operatorname{Tr}\left\{\frac{1}{2} F_{\mu \nu}^{2}+\left(\nabla_{\mu} X_{i}\right)^{2}+\frac{1}{2}\left[X_{i}, X_{j}\right]^{2}-\left(\frac{\kappa g^{2}}{4 \pi}\right)^{2} X_{i}^{2}\right\} \\
& +\frac{\kappa}{4 \pi} \operatorname{Tr}\left\{\epsilon^{\mu \nu \rho}\left(A_{\mu} \partial_{\nu} A_{\rho}+\frac{2}{3} i A_{\mu} A_{\nu} A_{\rho}\right)-\frac{i}{3} \epsilon^{i j k} X_{i}\left[X_{j}, X_{k}\right]\right\} .
\end{aligned}
$$

Due to the Chern-Simons term the vector field topologically gains mass $M=\left|\frac{\kappa g^{2}}{4 \pi}\right|$ which is the same mass as the adjoint scalars have. Note that in the limit of $\kappa \rightarrow 0$, which corresponds to the limit $\theta \rightarrow 0$ in the brane configuration, one obtains the $N=4$ supersymmetric pure Yang-Mills Lagrangian.

The Coulomb branch of supersymmetric gauge theory is described by solutions of the following flatness condition for the adjoint scalar fields

$$
M^{2} X_{i}-\frac{M}{2} i \epsilon_{i j k}\left[X_{j}, X_{k}\right]-\left[X_{j},\left[X_{i}, X_{j}\right]\right]=0 .
$$


For real $X_{i}$, the general solution of this condition is $\left\langle X_{i}\right\rangle=0$. So the Coulomb branch of this theory is completely lifted because of the mass term coming from the Chern-Simons interaction.

From the point of view of the brane configuration, it is easy to understand this Coulomb branch moduli as the following. In the $N=4$ brane configuration, $N_{c}$ D3-branes can freely moved on the $\left(x^{3}, x^{4}, x^{5}\right)$-space since two NS5-branes are parallel. So we can identify positions of D3-branes in the $\left(x^{3}, x^{4}, x^{5}\right)$-space with vevs of the three real adjoint scalar fields, which describe a part of the Coulomb branch.

However, in the $N=3$ configuration, since the left NS5-brane and the right $(p, q) 5$ brane is completely twisted in $\left(x^{3}, x^{4}, x^{5}\right)$ - and $\left(x^{7}, x^{8}, x^{9}\right)$-space, D3-branes can not move anywhere. This means that the corresponding vevs of the adjoint scalars must be at origin, namely, $\left\langle X_{i}\right\rangle=0$, which coincide with the solution of the flatness condition (3.3).

The mass of the adjoint scalars can be written in terms of the rotation angle of the $(p, q) 5$-brane as

$$
\left|\frac{\kappa g^{2}}{4 \pi}\right|=\frac{1}{4 \pi L}|\tan \theta|,
$$

where we use the relation (2.1) and (2.2). This relation also agree with the results in ref. 13, 14.

In the $\theta \rightarrow 0$ limit the mass of the adjoint scalar fields vanishes and at the same time the Chern-Simons interaction is dropped. In the brane configuration the right $(p, q) 5$-brane become NS5-brane and to be parallel to the left NS5. So we have $N=4$ supersymmetric pure Yang-Mills theory. On the other hand, in the limit $\theta \rightarrow \pi / 2$ the mass of the vector multiplet goes to infinity and all gauge degrees of freedom are decoupled. In this limit the right $(p, q) 5$-brane become D5-brane which extends in the $\left(x^{0}, x^{1}, x^{2}, x^{7}, x^{8}, x^{9}\right)$-space. This configuration also recover the $N=4$ supersymmetry again and appears in the Higgs branch of the Hanany-Witten configuration [1]].

\subsection{Breaking to $N=2$}

We next consider the supersymmetry breaking of $N=3$ theory down to $N=2$. In the brane language this is done by making two of the angles different from the others. Namely, we 
have the configuration

$$
\begin{aligned}
\text { NS5 } & :(012345) \\
N_{c} \times \text { D3 } & :(012|6|) \\
(p, q) 5 & :\left(012\left[\begin{array}{l}
3 \\
7
\end{array}\right]_{\psi}\left[\begin{array}{l}
4 \\
8
\end{array}\right]_{\psi}\left[\begin{array}{l}
5 \\
9
\end{array}\right]_{-\theta}\right) .
\end{aligned}
$$

As the result, the corresponding mass of the adjoint scalars become different.

We can field theoretically understand this supersymmetry breaking. Originally $N=3$ vector multiplet contains the fields $\left(A_{\mu}, \lambda_{i}, X_{i}, \chi\right)$. If we give the different mass $\mu$ to the $X_{1}, X_{2}$ and $\lambda_{3}, \chi$, these fields form the $N=2$ neutral chiral supermultiplet $\Phi$. The rests of the fields $\left(A_{\mu}, \lambda_{a}, X_{3}\right)$ are contents of the $N=2$ vector multiplet, which still have the mass $\left|\frac{\kappa g^{2}}{4 \pi}\right|$.

In this $N=2$ theory three adjoint scalars are massive as long as $\psi \neq 0$. So the Coulomb branch of this theory is still lifted and there is no moduli. This corresponds to the twisting configuration of the NS5-brane and the rotated $(p, q) 5$-brane. However, if we set $\psi=0$ the NS5-brane and $(p, q) 5$-brane become parallel in the $\left(x^{3}, x^{4}\right)$-space. Therefore D3-branes can now freely move in the $\left(x^{3}, x^{4}\right)$-space. In this configuration, two adjoint scalars $X_{1}$ and $X_{2}$ become massless and the Coulomb branch appears.

\section{Broken and Unbroken Phases of Abelian Theory}

In this section we consider the Higgs branches of the $N=2$ supersymmetric Chern-Simons theory. We first briefly review the description of the Higgs branch in the Hanany-Witten configuration. The inclusion of the matter hypermultiplets corresponds to the addition of the D5-branes, which extend in the $\left(x^{0}, x^{1}, x^{2}, x^{7}, x^{8}, x^{9}\right)$-space, between two NS5-branes. The matter hypermultiplets come from the open string stretched between D5-branes and D3-branes't. For simplicity, we consider only Abelian theory with one matter hypermultiplet, which includes two complex scalar fields $(q, \widetilde{q})$ from now on.

If we denote the distance between the D5-brane and the D3-brane in the $\left(x^{3}, x^{4}, x^{5}\right)$ -

\footnotetext{
${ }^{1}$ There is a couple of chiral fields with opposite chirality with respect to orientation of strings.
} 
space as $d$, the mass of the hypermultiplet is given by

$$
m=T_{\mathrm{F} 1} d=\frac{d}{2 \pi l_{s}^{2}}
$$

where $T_{\mathrm{F} 1}$ is the string tension and $l_{s}$ is the string length. In the decoupling limit of massive excitation of strings $l_{s} \rightarrow 0$, the quantity of $m$ must be fixed.

The Higgs branch of this theory emanates from the point where the hypermultiplet is massless, namely, $d=0$ in the brane configuration. When $d=0$, the D5-brane can separate the D3-brane into two parts. As the result, two NS5-branes can shift each other's positions in the $\left(x^{7}, x^{8}, x^{9}\right)$-space. We denote this difference as $\widetilde{d}$.

The gauge symmetry in this branch is completely broken since the D3-brane is divided into two parts by the D5-brane and all gauge degrees of freedom are decoupled. In the broken phase of the Abelian gauge theory, there exists a vortex state, which is an electricmagnetic dual object of the fundamental matter. Since the electric-magnetic duality of the brane effective theory is achieved by the Type IIB S-duality, the stringy counterpart of the vortex state is a D-string stretched between two separated parts of D3-brane. The mass of this state is

$$
\widetilde{m}=T_{\mathrm{D} 1} \widetilde{d}=\frac{\widetilde{d}}{2 \pi \widetilde{l}_{s}^{2}},
$$

where $\widetilde{l}_{s}=g_{s}{ }^{1 / 2} l_{s}$ is a string length of the dual theory. Filed theoretically, the mass of the vortex state is given by the Fayet-Iliopoulos (FI) parameter $\zeta$. If we define as

$$
\zeta=\frac{\widetilde{m}}{4 \pi}=\frac{\widetilde{d}}{8 \pi^{2} \widetilde{l}_{s}^{2}},
$$

the FI parameter is proportional to the difference of two NS5-brane positions in the $x^{9}$ direction.

Next, we consider a similar situation in the $N=2$ brane configuration with $(p, q) 5$ brane. We add only one set of massless matter superfields which plays a role of Higgs field and consider the Abelian case, that is, the Maxwell Chern-Simons Higgs system. Moreover, we set the angles $\psi$ to $\pi / 2$ in the $N=2$ brane configuration. So the brane configuration is as follows

NS5 : (012345) 


$$
\begin{aligned}
\text { D3 } & :(012|6|) \\
\text { D5 } & :(012789) \\
(p, q) 5 & :\left(01278\left[\begin{array}{l}
5 \\
9
\end{array}\right]_{-\theta}\right) .
\end{aligned}
$$

The two adjoint scalars $X_{1}$ and $X_{2}$ are decoupled from the theory and so we can easily pay attention to the true nature of the Higgs phase of the Abelian theory.

The squark Higgs fields $q, \widetilde{q}$ in the matter superfields couple to a non-dynamical Dfield in the off-shell vector multiplet. If we integrate out the auxiliary D-field we have a bosonic scalar potential

$$
V=-\frac{g^{2}}{2}\left(|q|^{2}-|\widetilde{q}|^{2}-\zeta+\frac{\kappa}{4 \pi} X_{3}\right)^{2}-X_{3}^{2}\left(|q|^{2}+|\widetilde{q}|^{2}\right)
$$

where $\zeta$ is the FI parameter.

The potential of the aforementioned Maxwell Higgs theory is obtained by setting $\kappa=X_{3}=0$. In this case there is only the asymmetric phase $|q|^{2}-|\widetilde{q}|^{2}=\zeta$. However, the potential (4.4) admits two distinct vacua, an asymmetric phase and a symmetric phase. We examine the correspondence between each branch and the brane configuration from now on.

\section{asymmetric phase}

The asymmetric phase is described by $|q|^{2}-|\widetilde{q}|^{2}=\zeta$ and $X_{3}=0$. This phase is the same as the asymmetric phase in the previous case. So the corresponding configuration is also very similar to the Maxwell Higgs one. Namely, the D5-brane divide the D3brane into two segments. One of the segments of the D3-brane stretch between the left NS5-brane and the D5-brane and the other is between the D5-brane and the right $(p, q) 5$ brane. In this phase the relative position of the 5 -branes along the $x^{9}$-direction can differ because of this split of the D3-brane and the gauge symmetry on the D3-brane is broken simultaneously. We denote the difference of 5 -branes in the $x^{9}$-direction as $\widetilde{d}$. (See Fig.11.)

There also exists the topological vortex state in this broken phase of the Maxwell Chern-Simons Higgs system. However, in the $(p, q) 5$-brane configuration we can not simply identify this vortex state with the D-string stretch between two fragments of the D3-brane. From the SUGRA solution of the $(p, q) 5$-brane, there is a non-trivial vev of 


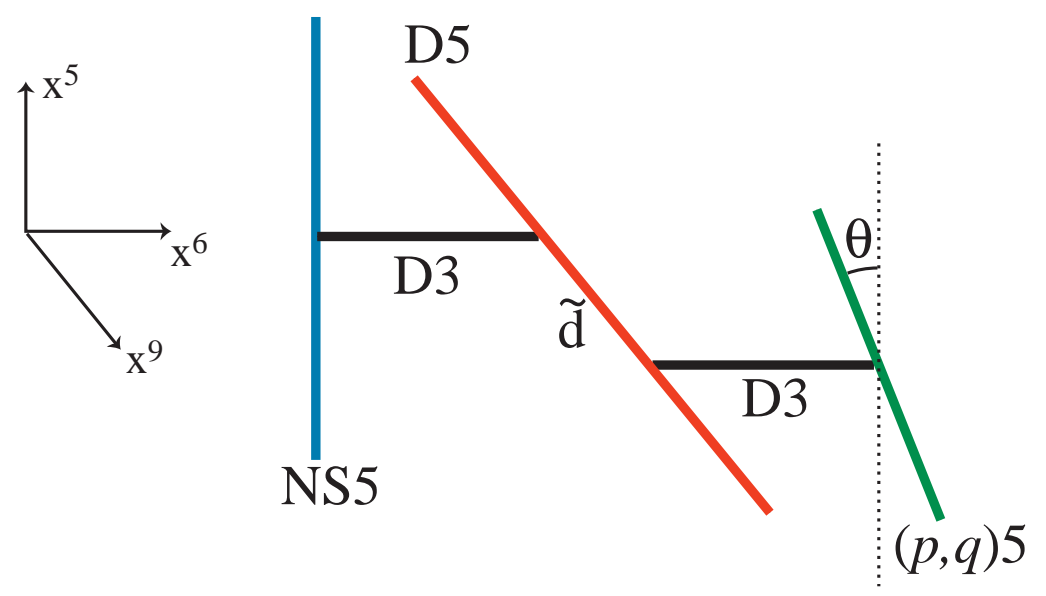

Figure 1: The brane configuration in the asymmetric phase.

the axion field like as [10]

$$
\chi\left(x^{6}, x^{7}, x^{8}, x^{9}\right)=\frac{1}{g_{s}} \frac{\sin \theta \cos \theta\left(H^{1 / 3}-H^{2 / 3}\right)}{H^{-1 / 3} \sin ^{2} \theta+H^{2 / 3} \cos ^{2} \theta},
$$

where $H=1+\frac{l_{s}^{2}}{r^{2}}$ is a harmonic function on the $\left(x^{6}, x^{7}, x^{8}, x^{9}\right)$-space and $r$ is a distance from the $(p, q) 5$-brane. We now assume that the distance $L$ between two 5 -branes is satisfied $L \ll l_{s} \rrbracket$. Since the D5-brane sits between two 5-branes, the distance from $(p, q) 5$-brane $r$ is also satisfied $r \ll l_{s}$. In this limit, that is, the axion field take a constant value near the D5-brane

$$
\chi=-\frac{1}{g_{s}} \tan \theta=-\kappa .
$$

In this background, D-string must appear as a dyon with electric charge proportional to $\chi$ by the Witten effect. In fact, since the tension of $(a, b)$-string in this background is given by 15

$$
T_{(a, b)}=\frac{1}{2 \pi l_{s}^{2}} \sqrt{\left(\frac{b}{g_{s}}\right)^{2}+(a+b \chi)^{2}},
$$

the D-string, i.e. $(0,1)$-string, is not lightest state. A lighter state is a $(\kappa, 1)$-string with the tension

$$
T_{(\kappa, 1)}=\frac{1}{2 \pi l_{s}^{2} g_{s}}
$$

\footnotetext{
${ }^{2}$ This limit is connected with the pure Chern-Simons limit $(\kappa$ is fixed and $g \rightarrow \infty$ ).
} 
which is the same tension as the D-string in the Maxwell theory configuration. Therefore the vortex state near the $(p, q) 5$-brane configuration is not only magnetically charged but also electrically charged.

Field theoretically, the Chern-Simons interaction gives the relation between electric charge $Q$ and magnetic flux $\Phi$

$$
Q=\kappa \Phi
$$

from the Gauss law constraint. So the vortex solution carry both magnetic charge and electric charge in the ratio of 1 to $\kappa$. This consequence exactly agree with the $(\kappa, 1)$-string state around the $(p, q) 5$-brane background.

\section{symmetric phase}

For the symmetric phase the vevs of the scaler fields are

$$
\begin{aligned}
& q=\widetilde{q}=0, \\
& X_{3}=4 \pi \zeta / \kappa .
\end{aligned}
$$

This phase does not exist in the Maxwell Higgs system. And also there is no corresponding configuration in the $N=4$ Hanany-Witten setup. However, in our case there exists another possible vacuum configuration for $\zeta \neq 0$ due to the rotation of the 5 -brane. If we want to set $\zeta \neq 0$ we must shift the positions of the 5 -branes each other along the $x^{9}$-direction. In general, the D3-brane suspended between the 5-branes prevents this motion. One of settlements is the division of the D3-brane into two parts as we mentioned before. Another solution is that the D3-brane slide up along the $x^{5}$-direction and is attached again on the intersecting point of the NS5-brane and the rotated $(p, q) 5$-brane in the $\left(x^{5}, x^{9}\right)$-plane. (See Fig. 2.)

In this symmetric phase configuration, the position of the D3-brane in the $x^{5}$ coordinate is $x^{5}=\widetilde{d} / \tan \theta$ where $\widetilde{d}$ is a gap of the 5 -brane positions. The gap $\widetilde{d}$ corresponds to the FI parameter $\zeta$ as in eq. (4.3) and the $x^{5}$ position of the D3-brane corresponds to the vev of the adjoint scalar $X_{3}$. So we find that the adjoint scalar vev is given by

$$
\begin{aligned}
X_{3} & =\frac{\tilde{d}}{2 \pi l_{s}^{2} \tan \theta} \\
& =\frac{4 \pi \zeta}{p / q} .
\end{aligned}
$$



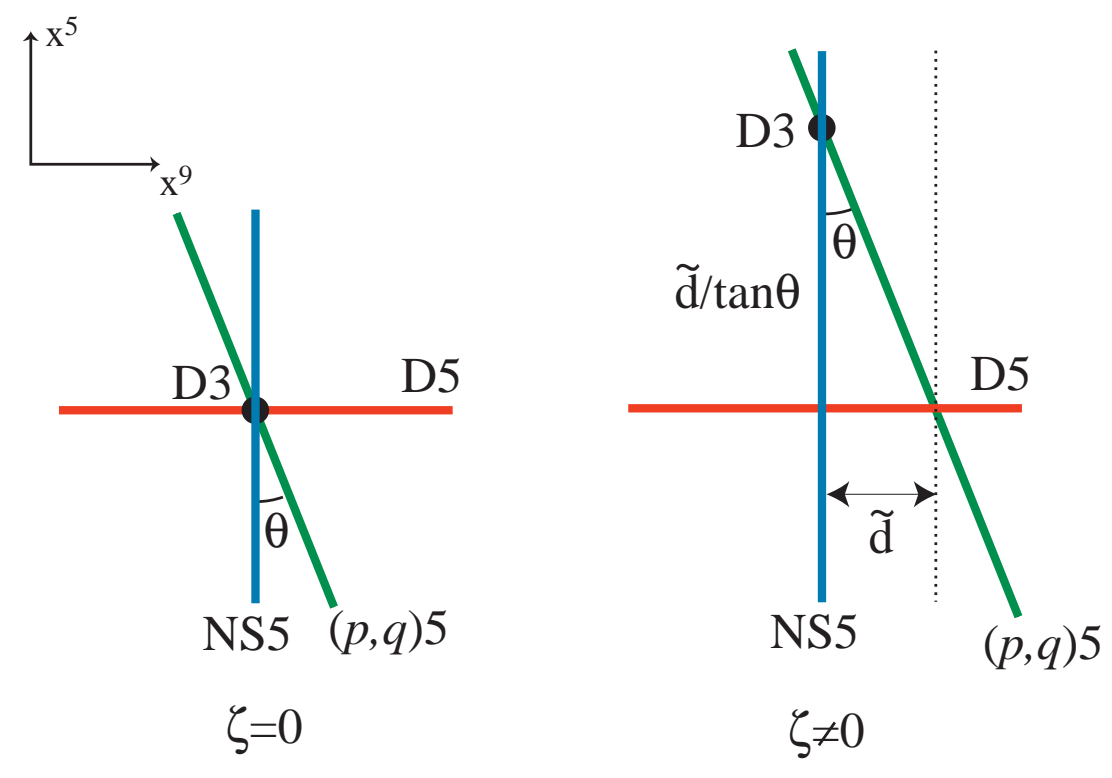

Figure 2: The brane configuration in the symmetric phase

This result is exactly agree with (4.11).

Moreover, the distance between the D3 and D5-brane is $\widetilde{d} / \tan \theta$. This means that the mass of the matter superfields which is coming from the fundamental string between D3 and D5-brane is $4 \pi \zeta / \kappa$. Correspondingly, when the $X_{3}$ field has the vev (4.11) the scalar potential (4.4) gives the mass $4 \pi \zeta / \kappa$ to the $q$ and $\widetilde{q}$. This is also consistent with the brane picture.

Finally, we would like to comment on vortex states in this phase. In this symmetric phase, it is known that there exists a non-topological vortex [16, 17]. However, since charges of the non-topological vortex contain a continuous parameter, it seems to be difficult to find the corresponding state in string theory.

\section{Acknowledgments}

I would like to thank T. Kitao and N. Ohta for arguments during the early stage of this work. I also grateful to B.-H. Lee for useful discussions and comments at Niseko Winter School. 


\section{References}

[1] A. Hanany and E. Witten, "Type IIB Superstrings, BPS Monopoles, And ThreeDimensional Gauge Dynamics," Nucl. Phys. B492 (1997) 152-190, hep-th/9611230.

[2] J. de Boer, K. Hori, Y. Oz and Z. Yin, "Branes and Mirror Symmetry in N=2 Supersymmetric Gauge Theories in Three Dimensions," Nucl. Phys. B502 (1997) 107-124, hep-th/9702154.

[3] J. de Boer, K. Hori and Y. Oz, "Dynamics of N=2 Supersymmetric Gauge Theories in Three Dimensions," Nucl. Phys. B500 (1997) 163-191, hep-th/9703100.

[4] A. Giveon and D. Kutasov, "Brane Dynamics and Gauge Theory," hep-th/9802067.

[5] A. Karch, "Field Theory Dynamics from Branes in String Theory," hep-th/9812072.

[6] J.P. Gauntlett, G.W. Gibbons, G. Papadopoulos and P.K. Townsend, "Hyper-Kahler manifolds and multiply-intersecting branes," Nucl. Phys. B500 (1997) 133-162, hep-th/9702202.

[7] B.M. Zupnik and D.G. Pak, "Topologically Massive Gauge Theories in Superspace," Sov. Phys. J. 31 (1988) 962-965; B.M. Zupnik and D.V. Khetselius, "ThreeDimensional Extended Supersymmetry in the Harmonic Superspace," Yad. Fiz. 47 (1988) 1147-1156.

[8] H.-C. Kao and K. Lee, "Self-Dual Chern-Simons Higgs Systems with an N=3 Extended Supersymmetry," Phys. Rev. D46 (1992) 4691-4697, hep-th/9205115.

[9] H.-C. Kao, "Self-Dual Yang-Mills Chern-Simons Higgs Systems with an N=3 Extended Supersymmetry," Phys. Rev. D50 (1994) 2881-2892.

[10] T. Kitao, K. Ohta and N. Ohta, "Three-Dimensional Gauge Dynamics from Brane Configurations with (p,q)-Fivebrane," Nucl. Phys. B539 (1999) 79-106, hep-th/9808111.

[11] A. Kapustin and M.J. Strassler, "On Mirror Symmetry in Three Dimensional Abelian Gauge Theories," hep-th/9902033. 
[12] N. Ohta and P. K. Townsend, "Supersymmetry of M-Branes at Angles," Phys. Lett. B418 (1998) 77-84, hep-th/9710129.

[13] S. Elitzur, A. Giveon and D. Kutasov, "Branes and N=1 Duality in String Theory," Phys. Lett. B400 (1997) 269-274, hep-th/9702014.

[14] J.L.F. Barbon, "Rotated Branes and N=1 Duality," Phys. Lett. B402 (1997) 59-63, hep-th/9703051.

[15] J.H. Schwarz, "An SL(2,Z) Multiplet of Type IIB Superstrings," Phys. Lett. B360 (1995) 13-18; Erratum-ibid. B364 (1995) 252 , hep-th/9508143.

[16] R. Jackiw and E. Weinberg, "Self-Dual Chern-Simons Vortices," Phys. Rev. Lett. 64 (1990) 2334.

[17] R. Jackiw, K. Lee and E. Weinberg, "Self-Dual Chern-Simons Solitons," Phys. Rev. D42 (1990) 3488. 